\title{
The contents of bibenzyl derivatives, flavonoids and a phenanthrene in selected Dendrobium spp. and the correlation with their antioxidant activity
}

\author{
Rattanathorn Choonong $^{\mathrm{a}, \mathrm{b}}$, Witcha Sermpradit ${ }^{\mathrm{a}, \mathrm{b}}$, Tharita Kitisripanya ${ }^{\mathrm{c}}$, Boonchoo Sritularak ${ }^{\mathrm{d}}$, \\ Waraporn Putalun ${ }^{\mathrm{a}, \mathrm{b}, *}$ \\ ${ }^{a}$ Faculty of Pharmaceutical Sciences, KhonKaen University, KhonKaen 40002 Thailand \\ b Research Group for Pharmaceutical Activities of Natural Products using Pharmaceutical Biotechnology \\ (PANPB), National Research University-KhonKaen University, KhonKaen 40002 Thailand \\ c Department of Pharmacognosy, Faculty of Pharmacy, Mahidol University, Bangkok 10400 Thailand \\ d Department of Pharmacognosy and Pharmaceutical Botany, Faculty of Pharmaceutical Sciences, \\ Chulalongkorn University, Bangkok 10330 Thailand
}

${ }^{*}$ Corresponding author, e-mail: waraporn@kku.ac.th

Received 11 Dec 2018

Accepted 30 Jun 2019

\begin{abstract}
Dendrobium spp. (the Orchidaceae family) is widely distributed in Asian countries. The fresh or dried stems of Dendrobium spp. have long been used in traditional Chinese medicine for their tonic and antipyretic properties. Bibenzyl derivatives (moscatilin, gigantol, crepidatin, and chrysotoxin), flavonoids (eriodictyol and homoeriodictyol) and phenanthrene lusianthridin are the major active compounds in the stems of Dendrobium spp. Most of these compounds have been recorded in these plants in Thailand; however, their concentrations have not yet been reported. Therefore, in this study, dried stems of selected Dendrobium spp. (33 species) in Thailand were extracted and analyzed by using high-performance liquid chromatography (HPLC). The HPLC method was performed on a reversed-phase column using a mobile phase gradient system of $20-32 \%$ acetonitrile in $1.5 \%$ acetic acid with a flow rate of $0.8 \mathrm{ml} / \mathrm{min}$ and a detection wavelength of $280 \mathrm{~nm}$. The HPLC method displayed good linearity with coefficients of determination $\left(R^{2}>0.997\right)$ for the seven active compounds analyzed. The variation in intra- and inter-day analysis (\%RSD) was less than $0.5 \%$. Additionally, the extracts of selected Dendrobium spp. were investigated for antioxidant activity by DPPH and FRAP radical scavenging assays. The results showed good activities in some species. The correlation between the seven active compounds and the antioxidant levels ( $\mathrm{IC}_{50}$ and FRAP values) were significant in bibenzyl derivatives and homoeriodictyol as determined by the Pearson correlation. In conclusion, the developed HPLC method can be used for the determination of active compounds in Dendrobium spp., which is useful for further studies of the species containing the highest levels of these compounds.
\end{abstract}

KEYWORDS: Dendrobium spp., bibenzyl derivatives, flavonoids, phenanthrene, high-performance liquid chromatography, antioxidant activity

\section{INTRODUCTION}

Dendrobium spp. (Orchidaceae family) are widely distributed in Asian countries. The fresh or dried stems of different species of Dendrobium, especially D. nobile (known as 'Shi-hu' or 'Huang-cao'), have been used in traditional Chinese medicine as tonics, antipyretics and for the treatment of diabetes ${ }^{1-3}$. In the Ayurvedic system of medicine in India, orchids are commonly used as drugs such as 'jewanti' (D. alpestre $)^{3}$. In the north and the northeastern parts of Thailand, Dendrobium species are grown in mountainous areas with a humid environment.
Many Dendrobium species have been recorded in the office of the forest herbarium of Thailand; however, the content of active compounds and their bioactivities have not yet been reported.

Bibenzyl derivatives (moscatilin, gigantol, crepidatin and chrysotoxin), flavonoids (eriodictyol and homoeriodictyol) and phenanthrene lusianthridin are the major active compounds that can be found in the stems of Dendrobium spp. ${ }^{4}$. Pharmacological studies have demonstrated that moscatilin has antimutagenic activity in human colorectal cancer cells and anti-angiogenesis activity was seen in vitro and in vivo tests ${ }^{5,6}$. Bibenzyl and phenanthrene 
compounds, such as gigantol, moscatilin and lusianthridin, exhibit anti-migratory activities and can inhibit filopodia formation in nonsmall cell lung cancer cells ${ }^{7-9}$. In addition, the antiplatelet aggregating activity of moscatilin, homoeriodictyol and gigantol was reported in an in vitro test ${ }^{10}$. Therefore, the analysis method for markers in Dendrobium spp. available in Thailand would provide information about which species contain the highest levels of bioactive markers. Implementation of high-performance liquid chromatography (HPLC) with mass spectrometry and diode array detection methods for the analysis of bioactive phenols in Dendrobium aurantiacum var. denneanum ${ }^{11}$ and Dendrobium spp. ${ }^{12}$ were reported.

In this study, the simultaneous determination of seven important markers by HPLC-UV was developed, and the levels of chemical markers from Dendrobium spp. and their antioxidant activities were evaluated for correlation. The results from this study will be useful for further studies in specified details.

\section{MATERIALS AND METHODS}

\section{Chemicals and reagents}

The reference compounds, bibenzyl derivatives (moscatilin, gigantol, crepidatin, chrysotoxin), flavonoids (eriodictyol and homoeriodictyol) and phenanthrene lusianthridin, were obtained from Dr. Boonchoo Sritularak in a previous study $^{7-9}$. 1,1-Diphenyl-2-picrylhydrazyl (DPPH), 2,4,6-tripyridyl-s-triazine (TPTZ), and 6-hydroxy2,5,7,8-tetramethylchromane-2-carboxylic acid (Trolox) were purchased from Sigma-Aldrich Chemical Co. (St. Louis, MO, USA). All other chemicals and reagents were of analytical grade.

\section{Plant materials}

A total of 33 samples of Dendrobium species were obtained from local markets in the Khon Kaen province and Bangkok, Thailand. The plant samples were selected based on their size relevant to 1 - to 2-year-old cultivated plants. All specimens were identified by Dr. Boonchoo Sritularak. Reference specimens (NI-PSKKU073-106) were deposited in the herbarium of the Faculty of Pharmaceutical Sciences, Khon Kaen University, Thailand.

\section{Sample preparation for HPLC analysis and antioxidant assays}

The stems of Dendrobium species were dried at $50{ }^{\circ} \mathrm{C}$ until achieving a constant weight and then ground into a powder. Dried powder was accurately weighed to $50 \mathrm{mg}$ per microcentrifuge tube, and $0.5 \mathrm{ml}$ of methanol was added. Ultrasonic-assisted extraction was done for $15 \mathrm{~min}$ at ambient temperature conditions. Then, the extract was centrifuged at $5000 \mathrm{rpm}$ for $5 \mathrm{~min}$. The supernatant was collected into a new tube. The extraction was repeated such that the powder was extracted three times. The combined supernatant was then evaporated to dryness at room temperature and redissolved in $1 \mathrm{ml}$ methanol for HPLC analysis and antioxidant assays.

\section{Chromatographic conditions}

The HPLC system was performed on a SHIMADZU i-Series. A RP-18 column (LichroCART, $250 \mathrm{~mm} \times 4 \mathrm{~mm}, 5 \mu \mathrm{m}$ particle size, Merck, Germany) was used. The mobile phase system consisted of a gradient elution starting at $20 \%$ acetonitrile in $1.5 \%$ aqueous acetic acid, which gradually increased to $32 \%$ acetonitrile in $30 \mathrm{~min}$, and was held at $32 \%$ acetonitrile for $30 \mathrm{~min}$. The flow rate was 0.8 and $1 \mathrm{ml} / \mathrm{min}$ for $0-30 \mathrm{~min}$ and $30-60 \mathrm{~min}$ of runtime, respectively. The system was set at a temperature of $20^{\circ} \mathrm{C}$. All solvents were filtered with a Whatman $0.45 \mu \mathrm{m}$ nylon filter prior to use, and the detection wavelength was set at $280 \mathrm{~nm}$. The sample solutions were filtered and injected $(20 \mu \mathrm{l}$ per injection) by an autosampler. The peak area and chromatograms of each sample were recorded and analyzed.

\section{Method validation}

A series of standard solutions were prepared by mixing moscatilin, gigantol, crepidatin, chrysotoxin, eriodictyol, homoeriodictyol and lusianthridin to obtain solutions with final concentrations of 100.00 , $50.00,25.00,12.50,6.25,3.13,1.56$, and 0.78 $\mu \mathrm{g} / \mathrm{ml}$ for all compounds. Intraday precision was evaluated on the same day as the calibration curve by injecting each concentration of standard solution in triplicate. The linearity of each standard curve was established by plotting the peak area versus the concentration. Interday precision was determined over 3 days by repeating triplicate injections (20 $\mu l$ per injection) for each concentration of standard solutions each day. Intraday and interday precision were reported as relative standard deviation (\%RSD). The correlation and the regression equations were calculated in Microsoft ExCEL 2010. The limits of detection (LOD) and limits of quantification (LOQ) were determined by calculating the average baseline noise with a signal 3 and 10 times higher than that of the baseline noise, respectively. 
The accuracy was evaluated by means of recovery studies. Dendrobium spp. sample solutions were spiked with known amounts of the mixed standards at three different concentration levels $(25 \%, 50 \%$, and $100 \%$ over the range of compound contents in Dendrobium spp. samples). Then, the spiked and unspiked samples were analyzed and the percentage of recovery was reported, which was calculated by comparing the added amount and the determined amount of each reference.

\section{DPPH radical-scavenging activity assay}

Dendrobium spp. extracts (33 species) were diluted by methanol to several concentrations: $0.31,0.63$, $1.25,2.5,5$, and $10 \mathrm{mg}$ dry wt./ml. Seven standard solutions were diluted to concentrations of 1.25 , $2.5,5,10,25,50,100,200,500$, and $1,000 \mu \mathrm{g} / \mathrm{ml}$ in methanol. The reaction mixtures in 96-well plates consisted of sample $(100 \mu \mathrm{l})$ and DPPH (100 $\mu l, 0.2 \mathrm{mM}$ ), which were both freshly prepared by dissolution in methanol. The mixture was kept in dark conditions for $30 \mathrm{~min}$ at room temperature. Then, the absorbance was measured at $495 \mathrm{~nm}$ against a blank (methanol). All determinations were performed in triplicate. The antioxidant activity was analyzed according to an established DPPH assay protocol with some modifications ${ }^{13,14}$. The results were expressed as $\mathrm{IC}_{50}$ values, which are the concentration required to affect the scavenging of $50 \%$ of $\mathrm{DPPH}$ radicals. The $\mathrm{IC}_{50}$ values were calculated from the relationship curve of scavenging activities (\%) versus concentrations of the respective sample.

\section{Ferric reducing antioxidant power (FRAP) assay}

Dendrobium spp. extracts (33 species) were diluted by methanol to concentrations of $0.31,0.63$, $1.25,2.50,5.00,6.25$, and $12.5 \mathrm{mg}$ dry wt. $/ \mathrm{ml}$. Seven standard solutions were diluted to concentrations of $2.5,5,10,25,50$, and $100 \mu \mathrm{g} / \mathrm{ml}$ in methanol. The working FRAP reagent was freshly prepared and used on the same day by mixing $300 \mathrm{mM}$ acetate buffer $(\mathrm{pH} 3.6), 10 \mathrm{mM}$ TPTZ (2,4,6-tripyridyl-s-triazine) in $40 \mathrm{mM}$ hydrochloric acid and $20 \mathrm{mM}$ ferric chloride $\mathrm{FeCl}_{3} \cdot 6 \mathrm{H}_{2} \mathrm{O}$ at a ratio of 10:1:1 (v/v/v). The working FRAP reagent $(270 \mu \mathrm{l})$ and sample solutions $(30 \mu \mathrm{l})$ were mixed in 96-well plates and kept at $37^{\circ} \mathrm{C}$ for $30 \mathrm{~min}$. The absorbance was measured at $595 \mathrm{~nm}$. A standard curve was performed using Trolox (6-hydroxy2,5,7,8-tetramethylchromane-2-carboxylic acid) at concentrations of $10,15.6,25,31.25,50$, and $62.5 \mu \mathrm{g} / \mathrm{ml}$. All determinations were performed
Table 1 Regression equation, correlation coefficients, limit of quantification (LOQ) and limit of detection (LOD) for standards determined by HPLC data analysis!

\begin{tabular}{lllccc}
\hline Cmp & Range & Regression equation & $R^{2}$ & LOD $^{*}$ & LOQ $^{*}$ \\
\hline 1 & $0.78-25$ & $y=70314 x+514$ & 0.9999 & 0.14 & 0.48 \\
2 & $0.78-25$ & $y=63321 x+2242$ & 0.9998 & 0.16 & 0.53 \\
3 & $6.25-100$ & $y=8493 x+4134$ & 0.9999 & 1.32 & 4.40 \\
4 & $0.78-25$ & $y=97306 x-12718$ & 0.9976 & 0.10 & 0.32 \\
5 & $1.56-50$ & $y=18508 x+13981$ & 0.9993 & 0.41 & 1.35 \\
6 & $6.25-100$ & $y=10943 x-29578$ & 0.9990 & 1.57 & 5.23 \\
7 & $6.25-100$ & $y=11987 x-40232$ & 0.9982 & 1.51 & 5.03 \\
\hline \multirow{7}{*}{ Cmp $=$ compound; Range = linearity range $(\mu \mathrm{g} / \mathrm{ml}) ;$} \\
" measured in $\mu \mathrm{g} / \mathrm{ml}$.
\end{tabular}

in triplicate. The results were analyzed by using a standard curve and expressed as the FRAP value (mg Trolox/g dry weight of sample) ${ }^{13}$.

\section{Statistical analysis}

The results of the HPLC and antioxidant assays are presented as the mean \pm standard deviation of triplicate independent experiments. The Pearson correlation was used to analyze the relationship of the test results. The significance levels of $p<0.05$ or $p<0.01$ were considered to indicate significance. All statistical analyses were performed using the SPSS Statistics software version 17.0.

\section{RESULTS}

To determine the contents of bibenzyl derivatives (moscatilin, gigantol, crepidatin, chrysotoxin), flavonoids (eriodictyol and homoeriodictyol) and phenanthrene lusianthridin in Dendrobium species, we developed an assay using the HPLC-UV method with optimum conditions for quantitation. The optimum condition for the determination of all seven standard compounds was using a gradient elution which started at $20 \%$ acetonitrile in $1.5 \%$ aqueous acetic acid and gradually increased to $32 \%$ acetonitrile over $30 \mathrm{~min}$. The column was then eluted with $32 \%$ acetonitrile for $30 \mathrm{~min}$. The flow rates were 0.8 and $1 \mathrm{ml} / \mathrm{min}$ for $0-30 \mathrm{~min}$ and 30-60 $\mathrm{min}$ of runtime, respectively. The HPLC chromatograms of standards are shown in Fig. 1(A). The retention times were 19.0, 28.2, 31.6, 32.8, 34.1, 42.8, and $45.9 \mathrm{~min}$ for eriodictyol (1), homoeriodictyol (2), moscatilin (3), lusianthridin (4), gigantol (5), chrysotoxin (6), and crepidatin (7), respectively. Linearity was established by constructing a calibration curve for each marker compound. The regression equation shows good correlation coefficients $\left(R^{2}>0.997\right)$ in each established linearity range of the seven standard compounds (Table 1). Moreover, 

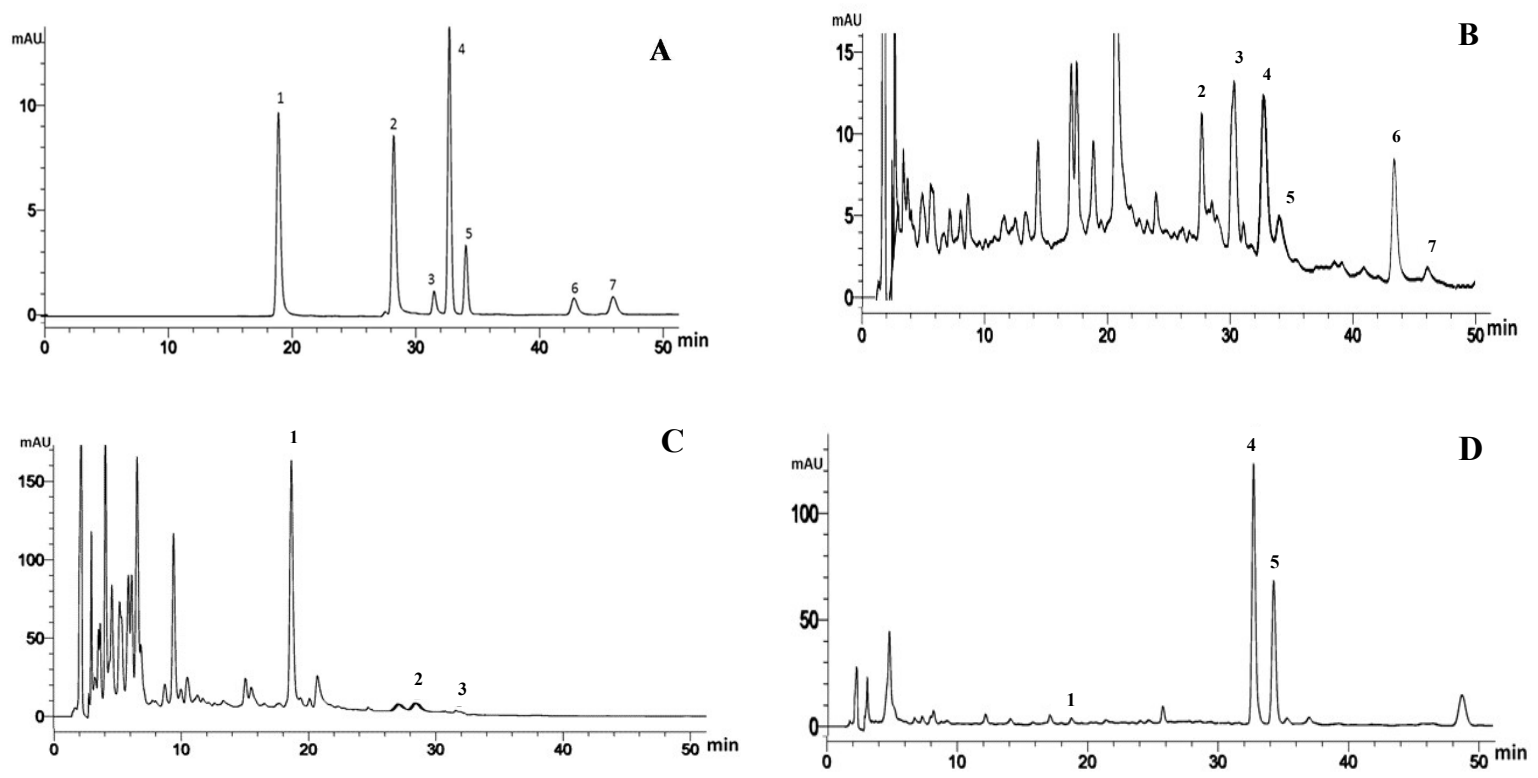

Fig. 1 HPLC Chromatograms (A) Standard compounds at a concentration of $3.13 \mu \mathrm{g} / \mathrm{ml}$ of (1) eriodictyol; (2) homoeriodictyol; (3) moscatilin; (4) lusianthridin; (5) gigantol; (6) chrysotoxin, and (7) crepidatin. (B) The methanol extract from the stem of D. fimbriatum; (C) D. ellipsophyllum, and (D) D. venustum.
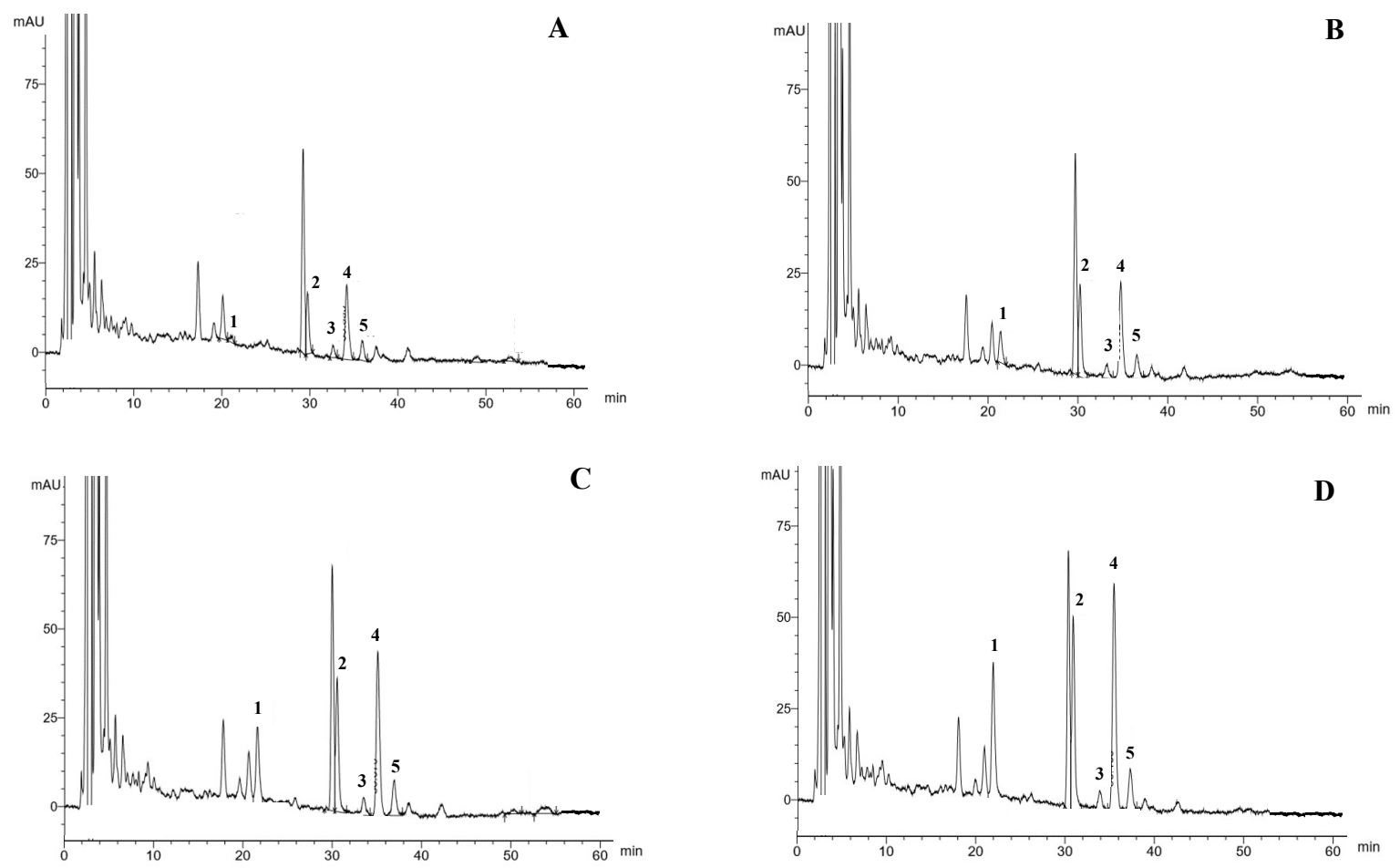

Fig. 2 A representative chromatogram of the recovery test (A) of the unspiked D. palpebrae methanolic extract and (B) the spiked samples of D. palpebrae methanolic extract at the concentration of $5 \mu \mathrm{g} / \mathrm{ml}$, (C) $10 \mu \mathrm{g} / \mathrm{ml}$, and (D) $20 \mu \mathrm{g} / \mathrm{ml}$ with (1) eriodictyol; (2) homoeriodictyol; (3) moscatilin; (4) lusianthridin; and (5) gigantol. 
Table 2 Recovery of standard compounds in Dendrobium spp. samples!

\begin{tabular}{lccc}
\hline Compound & $\begin{array}{c}\text { Spiked } \\
(\mu \mathrm{g} / \mathrm{ml})\end{array}$ & $\begin{array}{c}\text { Recovery } \\
(\%)\end{array}$ & $\begin{array}{c}\text { RSD } \\
(\%)\end{array}$ \\
\hline 1 & 5 & 102.46 & 0.84 \\
& 10 & 90.49 & 1.47 \\
& 20 & 91.03 & 2.80 \\
\hline 2 & 5 & 101.19 & 1.48 \\
& 10 & 90.93 & 2.29 \\
& 20 & 90.47 & 0.42 \\
\hline 3 & 5 & 103.26 & 1.11 \\
& 10 & 95.19 & 1.53 \\
& 20 & 97.76 & 4.15 \\
\hline 5 & 5 & 90.55 & 0.17 \\
& 10 & 90.57 & 0.56 \\
& 20 & 89.38 & 6.97 \\
\hline 6 & 5 & 96.91 & 1.97 \\
& 10 & 91.49 & 2.36 \\
& 20 & 92.99 & 1.75 \\
\hline 7 & 10 & 105.83 & 0.74 \\
& 20 & 95.33 & 2.32 \\
& 50 & 99.82 & 1.95 \\
\hline & 10 & 99.62 & 2.35 \\
& 20 & 90.69 & 4.79 \\
& 50 & 91.95 & 2.71 \\
\hline & & & \\
& 5 & &
\end{tabular}

${ }^{\dagger}$ Spiked $=$ spiked concentration.

Table $3 \quad \mathrm{IC}_{50}(\mathrm{mg} / \mathrm{ml})$ values determined by the DPPH assay and the antioxidant activity of seven compounds determined by the FRAP assay (mg Trolox/g dry wt.).

\begin{tabular}{lcc}
\hline Compound & IC $_{50}$ & FRAP value \\
\hline 1 & $18.16 \pm 0.15 \times 10^{-3}$ & $1066.46 \pm 24.51$ \\
2 & $583.88 \pm 9.40 \times 10^{-3}$ & $857.27 \pm 20.33$ \\
3 & $23.23 \pm 1.21 \times 10^{-3}$ & $2923.58 \pm 163.23$ \\
4 & $21.40 \pm 0.61 \times 10^{-3}$ & $1071.46 \pm 46.58$ \\
5 & $10.75 \pm 0.16 \times 10^{-3}$ & $2516.63 \pm 162.15$ \\
6 & $21.68 \pm 0.27 \times 10^{-3}$ & $1248.04 \pm 62.09$ \\
7 & $22.45 \pm 0.90 \times 10^{-3}$ & $1258.04 \pm 46.57$ \\
Trolox & $16.99 \pm 0.47 \times 10^{-3}$ & - \\
\hline
\end{tabular}

the limits of detection (LOD) and quantification (LOQ), which were respectively calculated as 3 and 10 times the signal-to-noise ratio of the smallest peak detected in chromatograms, are shown in Table 1. The intraday and interday assay precision values were analyzed by using the variation in measurement of the seven marker compounds. The relative standard deviations (\%RSD) of both intraday and interday were less than 5\% (data not
Table $4 \mathrm{IC}_{50}(\mathrm{mg} / \mathrm{ml})$, FRAP value (mg Trolox/g dry wt.) of various Dendrobium spp. extracts determined by the DPPH and FRAP assays, respectively.

\begin{tabular}{|c|c|c|}
\hline Name & $\mathrm{IC}_{50}$ & FRAP value \\
\hline D. albosanguineum & $0.93 \pm 0.06$ & $10.71 \pm 0.25$ \\
\hline D. anosmum & $5.84 \pm 0.27$ & $3.15 \pm 0.08$ \\
\hline D. bellatulum & $2.85 \pm 0.12$ & $11.80 \pm 0.38$ \\
\hline D. capilipes & $1.78 \pm 0.11$ & $11.51 \pm 0.68$ \\
\hline D. christyanum & $2.59 \pm 0.20$ & $7.75 \pm 0.40$ \\
\hline D. chrysotoxum & $3.34 \pm 0.27$ & $6.92 \pm 0.39$ \\
\hline D. cretaceum & $3.34 \pm 0.07$ & $7.66 \pm 0.75$ \\
\hline D. crumenatum & $3.95 \pm 0.14$ & $4.05 \pm 0.26$ \\
\hline D. delacourii & $1.65 \pm 0.17$ & $10.05 \pm 0.52$ \\
\hline D. draconis & $1.82 \pm 0.09$ & $5.86 \pm 0.22$ \\
\hline D. ellipsophyllum & $0.37 \pm 0.03$ & $25.96 \pm 0.84$ \\
\hline D. falconeri & $1.19 \pm 0.18$ & $11.15 \pm 0.74$ \\
\hline D. fimbriatum & $0.25 \pm 0.01$ & $53.02 \pm 1.58$ \\
\hline D. findlayanum & $1.28 \pm 0.07$ & $10.70 \pm 0.95$ \\
\hline D. formosum & $4.16 \pm 0.02$ & $5.16 \pm 0.31$ \\
\hline D. friedericksianum & $0.78 \pm 0.00$ & $19.62 \pm 1.07$ \\
\hline D. infundibulum & $2.39 \pm 0.02$ & $7.56 \pm 0.73$ \\
\hline D. kontumense & $2.68 \pm 0.23$ & $10.16 \pm 0.42$ \\
\hline D. lindleyi & $0.53 \pm 0.49$ & $9.06 \pm 0.29$ \\
\hline D. moschatum & $9.20 \pm 0.10$ & $2.66 \pm 0.20$ \\
\hline D. ochreatum & $0.83 \pm 0.06$ & $10.94 \pm 0.81$ \\
\hline D. pachyglossum & $1.10 \pm 0.12$ & $12.14 \pm 0.56$ \\
\hline D. palpebrae & $0.88 \pm 0.01$ & $16.01 \pm 0.77$ \\
\hline D. parishii & $0.36 \pm 0.01$ & $14.82 \pm 0.25$ \\
\hline D. primulinum & $0.88 \pm 0.02$ & $23.39 \pm 1.11$ \\
\hline D. pulchellum & $4.92 \pm 0.06$ & $6.01 \pm 0.25$ \\
\hline D. secundum & $4.46 \pm 0.23$ & $5.51 \pm 0.35$ \\
\hline D. secundum var. alba & $3.15 \pm 0.10$ & $5.91 \pm 0.25$ \\
\hline D. signatum & $1.16 \pm 0.23$ & $9.55 \pm 0.29$ \\
\hline D. soabriligue & $2.59 \pm 0.09$ & $11.17 \pm 0.62$ \\
\hline D. thyrsiflorum & $2.26 \pm 0.04$ & $8.25 \pm 0.73$ \\
\hline D. tortile & $0.95 \pm 0.02$ & $16.34 \pm 0.62$ \\
\hline D. venustum & $4.25 \pm 0.02$ & $26.02 \pm 1.14$ \\
\hline
\end{tabular}

shown). The amount of each standard added was 5, 10, and $20 \mu \mathrm{g} / \mathrm{ml}$. The standard compound recovery of spiked Dendrobium extract was tested to establish accuracy. After the extract was spiked with different concentrations of the seven standard compounds, the concentration was determined and calculated as a percentage of recovery, which presented good accuracy within $80-120 \%$ (Table 2). For example, the unspiked chromatogram of palpebrae methanolic extract is shown in Fig. 2(A). The spiked samples with (1) eriodictyol, (2) homoeriodictyol, (3) moscatilin, (4) lusianthridin, and (5) gigantol at concentrations of 5,10 , and $20 \mu \mathrm{g} / \mathrm{ml}$ are shown in Fig. 2(B,C,D). respectively. The contents of the bibenzyl derivatives moscatilin (3), gigantol (5), crepidatin (6), and chrysotoxin (7); the 
Table 5 Contents of total bibenzyl derivatives (moscatilin, gigantol, chrysotoxin, crepidatin), total flavonoids (eriodictyol, homoeriodictyol) and phenanthrene lusianthridin in stems from thirty-three Dendrobium spp.

\begin{tabular}{|c|c|c|c|c|c|c|c|c|c|}
\hline \multirow{2}{*}{$\begin{array}{l}\text { Dendrobium } \\
\text { spp. }\end{array}$} & \multicolumn{9}{|c|}{ Content (mg/g dry wt.) } \\
\hline & Mos. (3) & Gig.(5) & Chr.(6) & Cre.(7) & Bibenzyl & Eri.(1) & Hom.(2) & Flavonoids & Lus.(4) \\
\hline albosanguineum & $0.96 \pm 0.02$ & $0.14 \pm 0.00$ & $1.13 \pm 0.02$ & - & $2.23 \pm 0.05$ & - & - & - & - \\
\hline anosmum & $0.24 \pm 0.00$ & - & - & - & $0.24 \pm 0.00$ & $0.19 \pm 0.00$ & - & $0.19 \pm 0.00$ & - \\
\hline bellatulum & $0.11 \pm 0.01$ & $0.21 \pm 0.01$ & - & - & $0.32 \pm 0.02$ & - & - & - & $0.10 \pm 0.00$ \\
\hline capilipes & - & $0.67 \pm 0.05$ & - & - & $0.67 \pm 0.05$ & $0.07 \pm 0.00$ & - & $0.07 \pm 0.00$ & - \\
\hline christyanum & - & $0.07 \pm 0.00$ & - & - & $0.07 \pm 0.00$ & $0.02 \pm 0.00$ & - & $0.02 \pm 0.00$ & $0.06 \pm 0.00$ \\
\hline chrysotoxum & $0.34 \pm 0.01$ & - & - & - & $0.34 \pm 0.01$ & - & $0.09 \pm 0.00$ & $0.09 \pm 0.00$ & - \\
\hline cretaceum & $1.09 \pm 0.05$ & $0.18 \pm 0.01$ & - & - & $1.27 \pm 0.06$ & - & - & - & - \\
\hline crumenatum & - & $0.09 \pm 0.00$ & - & - & $0.09 \pm 0.00$ & - & - & - & $0.09 \pm 0.00$ \\
\hline delacourii & - & $0.99 \pm 0.03$ & - & - & $0.99 \pm 0.03$ & - & $0.05 \pm 0.00$ & $0.05 \pm 0.00$ & $0.74 \pm 0.03$ \\
\hline draconis & $0.14 \pm 0.00$ & $0.15 \pm 0.00$ & - & - & $0.29 \pm 0.01$ & $0.03 \pm 0.00$ & - & $0.03 \pm 0.00$ & - \\
\hline ellipsophyllum & $0.17 \pm 0.00$ & - & - & - & $0.17 \pm 0.00$ & $1.86 \pm 0.04$ & $0.07 \pm 0.00$ & $1.93 \pm 0.04$ & - \\
\hline falconeri & $0.13 \pm 0.01$ & - & $2.09 \pm 0.14$ & - & $2.22 \pm 0.15$ & - & - & - & - \\
\hline fimbriatum & $3.57 \pm 0.30$ & $0.35 \pm 0.01$ & $3.02 \pm 0.19$ & $0.43 \pm 0.01$ & $7.36 \pm 0.50$ & - & $0.24 \pm 0.01$ & $0.24 \pm 0.01$ & $0.22 \pm 0.02$ \\
\hline findlayanum & - & - & $0.53 \pm 0.02$ & $1.31 \pm 0.02$ & $1.85 \pm 0.04$ & - & $0.08 \pm 0.00$ & $0.08 \pm 0.00$ & - \\
\hline formosum & $0.58 \pm 0.02$ & $0.09 \pm 0.01$ & - & - & $0.67 \pm 0.03$ & $0.02 \pm 0.00$ & $0.13 \pm 0.01$ & $0.16 \pm 0.01$ & - \\
\hline friedericksianum & - & $0.07 \pm 0.00$ & $1.04 \pm 0.01$ & $0.31 \pm 0.00$ & $1.42 \pm 0.02$ & $0.20 \pm 0.00$ & $0.08 \pm 0.00$ & $0.28 \pm 0.00$ & - \\
\hline infundibulum & - & - & - & - & - & - & $0.02 \pm 0.00$ & $0.02 \pm 0.00$ & - \\
\hline kontumense & - & $1.04 \pm 0.07$ & - & - & $1.04 \pm 0.07$ & $0.02 \pm 0.00$ & - & $0.02 \pm 0.00$ & - \\
\hline lindleyi & - & - & - & - & - & - & - & - & $0.06 \pm 0.00$ \\
\hline moschatum & - & - & - & - & - & $0.06 \pm 0.00$ & - & $0.06 \pm 0.00$ & - \\
\hline ochreatum & $1.22 \pm 0.06$ & $0.08 \pm 0.00$ & - & - & $1.30 \pm 0.06$ & - & - & - & - \\
\hline pachyglossum & $0.75 \pm 0.00$ & $0.14 \pm 0.01$ & - & - & $0.89 \pm 0.01$ & $0.09 \pm 0.00$ & - & $0.09 \pm 0.00$ & - \\
\hline palpebrae & $0.87 \pm 0.01$ & $0.45 \pm 0.01$ & $0.32 \pm 0.00$ & $0.29 \pm 0.00$ & $1.93 \pm 0.02$ & $0.27 \pm 0.00$ & $0.99 \pm 0.01$ & $1.26 \pm 0.01$ & $0.25 \pm 0.01$ \\
\hline parishii & $2.36 \pm 0.07$ & - & - & - & $2.36 \pm 0.07$ & $0.10 \pm 0.00$ & $0.03 \pm 0.00$ & $0.13 \pm 0.00$ & - \\
\hline primulinum & $0.99 \pm 0.03$ & $1.01 \pm 0.02$ & - & - & $2.01 \pm 0.04$ & - & - & - & - \\
\hline pulchellum & $0.18 \pm 0.00$ & $0.02 \pm 0.00$ & - & - & $0.20 \pm 0.00$ & - & - & - & - \\
\hline secundum & - & - & - & - & - & - & - & - & - \\
\hline secundum var. alb & - & - & - & - & - & - & - & - & - \\
\hline signatum & - & - & $0.59 \pm 0.02$ & - & $0.59 \pm 0.02$ & $0.18 \pm 0.00$ & $0.25 \pm 0.00$ & $0.43 \pm 0.01$ & - \\
\hline soabriligue & $0.15 \pm 0.01$ & $0.50 \pm 0.01$ & - & - & $0.65 \pm 0.02$ & $0.03 \pm 0.00$ & $0.02 \pm 0.00$ & $0.24 \pm 0.00$ & $0.06 \pm 0.00$ \\
\hline thyrsiflorum & $0.70 \pm 0.03$ & $0.28 \pm 0.01$ & - & - & $0.98 \pm 0.04$ & - & $0.28 \pm 0.02$ & $0.28 \pm 0.02$ & $0.07 \pm 0.00$ \\
\hline tortile & - & - & $0.54 \pm 0.02$ & - & $0.54 \pm 0.02$ & $0.09 \pm 0.00$ & $0.09 \pm 0.00$ & $0.18 \pm 0.00$ & - \\
\hline venustum & - & $4.13 \pm 0.24$ & - & - & $4.13 \pm 0.24$ & $0.04 \pm 0.00$ & - & $0.04 \pm 0.00$ & $1.29 \pm 0.04$ \\
\hline
\end{tabular}

$-=$ not detected.

Table 6 Correlation analysis of marker compounds in Dendrobium spp. and antioxidant activities.

\begin{tabular}{clccccccccc}
\hline & \multicolumn{9}{c}{ Assay } & \multicolumn{7}{c}{ Compounds } \\
\cline { 3 - 11 } & & IC $_{50}$ & FRAP & 1 & 2 & 3 & 4 & 5 & 6 & 7 \\
\hline \multirow{3}{*}{ IC $_{50}$} & Pearson & 1 & $-.566^{* *}$ & -.193 & $-.206^{* *}$ & $-.315^{* *}$ & -.191 & -.197 & $-.339^{* *}$ & $-.204^{* *}$ \\
& Sig.(2-tailed) & - & .000 & .056 & .041 & .002 & .058 & .051 & .001 & .043 \\
& $\mathrm{~N}$ & 99 & 99 & 99 & 99 & 99 & 99 & 99 & 99 & 99 \\
\hline \multirow{4}{*}{ FRAP } & Pearson & $-.566^{* *}$ & 1 & $.264^{* *}$ & $.225^{*}$ & $.643^{* * *}$ & $.319^{* *}$ & $.326^{* *}$ & $.642^{* * *}$ & $.261^{* * *}$ \\
& Sig.(2-tailed) & .000 & - & .008 & .025 & .000 & .001 & .001 & .000 & .009 \\
& $\mathrm{~N}$ & 99 & 99 & 99 & 99 & 99 & 99 & 99 & 99 & 99 \\
\hline
\end{tabular}

"Correlation is significant at the 0.05 level (2-tailed); "* correlation is significant at the 0.01 level (2-tailed).

flavonoids eriodictyol (1) and homoeriodictyol (2); and phenanthrene lusianthridin (4) in 33 species of Dendrobium are shown in Table 5. The results showed that different species of Dendrobium had different chemical contents and characterizations. The seven standard markers were determined in all samples, and the concentration in $\mathrm{mg} / \mathrm{g}$ dry weight was calculated. The results show that $D$. fimbriatum contained the highest bibenzyl derivative content $(7.36 \pm 0.50 \mathrm{mg} / \mathrm{g}$ dry wt.) and the chromatogram is shown in Fig. 1(B). D. ellipsophyllum contained the highest flavonoid content $(1.93 \pm 0.04 \mathrm{mg} / \mathrm{g}$ dry wt.) and the chromatogram is shown in Fig. 1(C). The highest phenanthrene content $(1.29 \pm 0.04 \mathrm{mg} / \mathrm{g}$ dry wt.) was found in $D$. venustum, as seen in the chromatogram in Fig. 1(D).

From this study, 28 of the 33 Dendrobium spp. tested primarily contain compounds in the bibenzyl group, which indicates that the bibenzyl group can be used as a marker for Dendrobium spp. Our results 
showed that 31 species contained at least one of seven compounds, which can be used to identify the chemical constituents of each species. However, detection of any of the seven standard compounds in $D$. secundum and $D$. secundum var. alba was not achieved.

The DPPH and FRAP assays are the basic methods for screening the antioxidant activity in plant samples, which are usually reported as $\mathrm{IC}_{50}$ values and in $\mathrm{mg}$ Trolox/g dry weight, respectively. Hence, both assays were used to determine the antioxidant activity of seven marker compounds in 33 species of Dendrobium. The results are shown in Tables 3 and 4 respectively. Consequently, a Pearson correlation was used to analyze the significant correlation between the content of each marker compound in Dendrobium spp. and their antioxidant activities.

In the DPPH assay, the contents of moscatilin and chrysotoxin showed a significant correlation with the antioxidant activity at the significance level $p<0.01$, and the content of homoeriodictyol and crepidatin exhibited a significant correlation with antioxidant activity at $p<0.05$. In the FRAP assay, the contents of eriodictyol, moscatilin, lusianthridin, gigantol, chrysotoxin and crepidatin were significantly correlated with the antioxidant activity $(p<0.01)$. The content of homoeriodictyol was also significantly correlated $(p<0.05)$, as shown in Table 6.

\section{DISCUSSION}

The developed HPLC method is useful for analyzing the seven marker compounds in Dendrobium spp. According to the analysis by the Pearson correlation, Dendrobium spp. which contained high levels of bibenzyl groups can be predicted to possess high antioxidant activities. Based on our results, antioxidant activities from the DPPH and FRAP assays of homoeriodictyol, moscatilin, crepidatin and chrysotoxin exhibit good relationships with their contents $(p<0.05)$. These results were also related to the summed bibenzyl derivative group concentration in Dendrobium spp. such as D. fimbriatum, which contains the highest amount of total bibenzyl derivatives $(7.36 \pm 0.50 \mathrm{mg} / \mathrm{g})$ and exhibits the highest antioxidant activities in the DPPH and FRAP assays $\left(\mathrm{IC}_{50} 0.25 \pm 0.01 \mathrm{mg} / \mathrm{ml}\right.$ and $53.02 \pm 1.58 \mathrm{mg}$ Trolox/g dry wt., respectively). Therefore, bibenzyl contents can be used as antioxidant markers for Dendrobium species. In a previous study, bibenzyl groups in $D$. nobile also showed high antioxidant activity $^{15}$. These antioxidant activities conform to the traditional Chinese medicine 'Shi Hu', which is derived from the dried or fresh stems of several Dendrobium species and is widely used for the treatment of various diseases associated with the metabolic disorders of reactive oxygen species in the human body, such as chronic atrophic gastritis, diabetes, skin aging, and cardiovascular disease ${ }^{16}$.

Although flavonoids and phenanthrene have shown similarly high levels of antioxidant activity as the bibenzyl group, their contents only have a significant correlation $(p<0.05)$ in the FRAP assay, with the exception of homoeriodictyol, which showed a significant correlation in both the DPPH and FRAP assays. Hence, in further studies, other biological activity assays might be performed to ensure that key marker compounds can be used as bioactive markers for Dendrobium spp.

Acknowledgements: This work was supported by funding from The Thailand Research Fund (IRN61W0005) and the Faculty of Pharmaceutical Sciences, Khon Kaen University, Thailand.

\section{REFERENCES}

1. Xu J, Han Q, Li S, Chen X,Wang X, Zhao Z, Chen $\mathrm{H}$ (2013) Chemistry, bioactivity and quality control of Dendrobium, a commonly used tonic herb in traditional Chinese medicine. Phytochem Rev 12, 341-367.

2. Gutierrez RMP (2010) Orchids: A review of uses in traditional medicine, its phytochemistry and pharmacology. J Med Plants Res 4, 592-638.

3. Hossain MM (2011) Therapeutic orchids: traditional uses and recent advances - An overview. Fitoterapia 82, 102-140.

4. Lam Y, Ng TB, Yao RM, Shi J, Xu K, Sze SC, Zhang KY (2015) Evaluation of chemical constituents and important mechanism of pharmacological biology in Dendrobium Plants. Evid Based Complement Alternat Med 2015, 841752.

5. Chen TH, Pan SL, Guh JH, Liao CH, Huang DY, Chen CC, Teng CM (2008) Moscatilin induces apoptosis in human colorectal cancer cells: a crucial role of cJun $\mathrm{NH}_{2}$-terminal protein kinase activation caused by tubulin depolymerization and DNA damage. Clin Cancer Res 14, 4250-4258.

6. Tsai AC, Pan SL, Liao CH, Guh JH, Wang SW, Sun HL, Liu YN, Chen CC et al (2010) Moscatilin, a bibenzyl derivative from the India orchid Dendrobrium loddigesii, suppresses tumor angiogenesis and growth in vitro and in vivo Cancer Lett 292, 163-170.

7. Charoenrungruang S, Chanvorachote P, Sritularak B, Pongrakhananon V (2014) Gigantol, a bibenzyl from Dendrobium draconis, inhibits the migratory behavior of non-small cell lung cancer cells. J Nat Prod 77, 1359-1366. 
8. Klongkumnuankarn P, Busaranon K, Chanvorachote P, Sritularak B, Jongbunprasert V, Likhitwitayawuid K (2015) Cytotoxic and antimigratory activities of phenolic compounds from Dendrobium brymerianum. Evid Based Complement Alternat Med 2015, 350410.

9. Tanagornmeatar K, Chaotham C, Sritularak B, Likhitwitayawuid K, Chanvorachote P (2014) Cytotoxic and anti-metastatic activities of phenolic compounds from Dendrobium ellipsophyllum. Anticancer Res 34, 6573-6579.

10. Fan C, Wang W, Wang Y, Qin G, Zhao W (2001) Chemical constituents from Dendrobium densiflorum. Phytochemistry 57, 1255-1258.

11. Yang L, Wang Y, Zhang G, Zhang F, Zhang Z, Wang $\mathrm{Z}, \mathrm{Xu} \mathrm{L}$ (2007) Simultaneous quantitative and qualitative analysis of bioactive phenols in Dendrobium aurantiacum var. denneanum by high-performance liquid chromatography coupled with mass spectrometry and diode array detection. Biomed Chromatogr 21, 687-694.
12. Yang L, Wang Z, Xu L (2006) Simultaneous determination of phenols (bibenzyl, phenanthrene, and fluorenone) in Dendrobium species by high-performance liquid chromatography with diode array detection. $J$ Chromatogr A 1104, 230-237.

13. Yang H, Dong Y, Du H, Shi H, Peng Y, Li X (2011) Antioxidant compounds from propolis collected in Anhui, China. Molecules 16, 3444-3455.

14. Cherdshewasart W, Sutjit W (2008) Correlation of antioxidant activity and major isoflavonoid contents of the phytoestrogen-rich Pueraria mirifica and Pueraria lobata tubers. Phytomedicine 15, 38-43.

15. Zhang $\mathrm{X}, \mathrm{Xu} \mathrm{JK}$, Wang J, Wang NL, Kurihara H, Kitanaka S, Yao XS (2007) Bioactive bibenzyl derivatives and fluorenones from Dendrobium nobile. $J$ Nat Prod 70, 24-28.

16. Wang KZ, Gao WY (1997) Progress of Dendrobium medical plants research. Chin Tradit Herb Drugs 10, 633-635. 